\title{
Commentary on unique interstitial miRNA signature drives fibrosis in a murine model of autosomal dominant polycystic kidney disease.
}

\author{
Ameya Patil \\ Medical College of Wisconsin, Milwaukee, Wisconsin United States.
}

\section{Commentary}

Autosomal Dominant Polycystic Kidney Disease (ADPKD), the most common renal genetic disease, is characterized by formation and progressive enlargement of fluid-filled cysts [1]. A dynamic Peri-cystic Local Micro-environment (PLM) created between the cysts becomes fibrotic over time. Due to progressive fibrosis, over $50 \%$ of ADPKD patients will reach End-stage Renal Disease (ESRD) requiring renal replacement therapy. Despite this connection between fibrosis and ESRD, there is no Food and Drug Administration (FDA)-approved therapy targeting fibrosis in ADPKD.

In ADPKD, inflammation in PLM areas is consistently reported in human and animal models of PKD [2]. Both initiation and progression of PLM fibrosis requires an intricate network of communication between various cells types present in the PLM and cystic epithelial cells. Profibrogenic signals in the PLM can be dissected with the help of miRNA expression studies to develop targeted interventions. Such studies require precise sampling of PLM tissue which isolates local pathophysiology. Laser Capture Micro-dissection (LCM) offers an attractive strategy for such precise sampling.

We employed LCM to analyze the miRNA profile of PLM at 3 critical stages in our unique murine hypomorphic model of ADPKD:

1) Postnatal day (PN) 21, (prior to any fibrosis);

2) PN 28 (initiation of fibrosis); and

3) PN 42 (progression of fibrosis).

These results were compared to age-matched miRNA profiles of the whole kidney. A striking finding was that the expression of miRNAs in the PLM was very different than that of the whole kidney [2]. Published transcriptomic studies in whole kidneys have discovered critical renal miRNAs with abnormal expression that affect a wide range of cellular processes. Further, miRNAs have been reported to play a central role in the pathogenesis of ADPKD [3]. A critical limitation to whole kidney transcriptomic approaches is the kidney's cellular complexity: arrays from whole kidneys reflect the composite expression of 26 different cell types [4]. This limitation is exacerbated in disease when inflammatory cells invade the kidney. Decreased Pkdl expression leads to up regulation of Mcp1, which then promotes macrophage accumulation very close to the cyst wall in PLM [5]. These macrophages can undergo phenotypic changes (alternative activation) that accelerate disease. Recent studies demonstrate that miRNAs play a role in transcriptional regulation of such change in macrophage phenotype [6].
Accepted on October 29, 2018

Accumulating evidence shows that aberrantly expressed miRNAs play important roles in renal pathologies including fibrosis, and underscores their roles as potential targets for therapeutic intervention. However, the study of miRNA expression in vivo requires precise sampling in order to capture the specific cells that reflect the pathology of interest. The recent study of Patil et al. [2] highlights the pitfalls of failing to accurately and precisely sample appropriate cells for such analysis, and identifies unique miRNAs which may drive fibrosis in ADPKD. This study clearly demonstrates the error in relying on whole kidney samples for analysis of miRNAs to identify well-localized pathophysiological processes in ADPKD. Such analyses may lead one to incorrectly identify and therapeutically target inappropriate miRNAs or proteins (which could potentially exacerbate the disease). Therefore, published data that relies upon whole kidney transcriptomic analysis should be viewed with careful skepticism.

\section{References}

1. Harris PC, VE Torres. Polycystic kidney disease. Annu Rev Med. 2009;60:321-37.

2. Patil A, Avner ED, Pan CG, et al. Unique interstitial miRNA signature drives fibrosis in a murine model of autosomal dominant polycystic kidney disease. World J Nephrol. 2018;7(5):108-16.

3. Noureddine L, Hajarnis S, Patel V. MicroRNAs and Polycystic Kidney Disease. Drug Discov Today Dis Models. 2013;10(3):e137-e1743.

4. Al-Awqati Q, Oliver JA. Stem cells in the kidney. Kidney Int. 2002;61(2):387-95.

5. Cassini MF, Kakade VR, Kurtz E, et al. Mcp1 Promotes Macrophage-Dependent Cyst Expansion in Autosomal Dominant Polycystic Kidney Disease. J Am Soc Nephrol. 2018;29(10):2471-81.

6. Li H, Jiang T, Li MQ, et al. Transcriptional Regulation of Macrophages Polarization by MicroRNAs. Front Immunol. 2018;9:1175.

\section{*Correspondence to}

Ameya Patil

Assistant Professor

Pediatric Nephrology

Medical College of Wisconsin

Children's Hospital of Wisconsin

Ph: Work- 4143377140

E-mail: appatil@mcw.edu 